\title{
Gram-scale Synthesis and Multifunctional Properties of a Two-Dimensional Layered Copper (II) Coordination Polymer
}

Sachin Ashok Bhat ${ }^{\mathrm{a}, \mathrm{b}}$, Nani Babu Palakurthy, Nagaiah Kambhala, Angappane Subramanian", Doddamane S. Shankar Rao a , Subbarao Krishna Prasad ${ }^{\text {a }}$ and

Channabasaveshwar. V. Yelamaggad ${ }^{\text {a* }}$

\footnotetext{
${ }^{a}$ Centre for Nano and Soft Matter Sciences (CeNS), Jalahalli, Bengaluru 560 013, India.

${ }^{b}$ Department of Chemistry, Mangalore University, Mangalagangotri 574 199, India

* Corresponding Author: E-mail - yelamaggad@,cens.res.in
} 


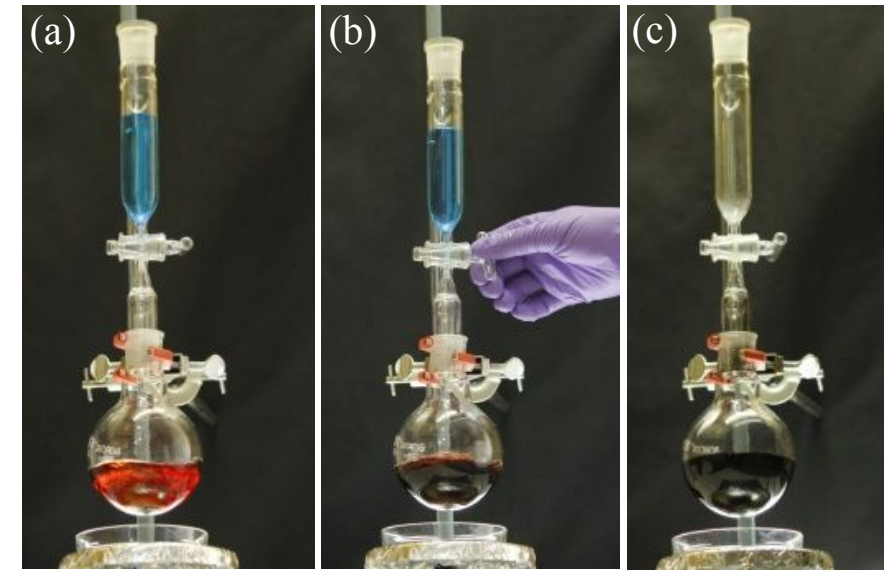

Figure S1. Photographs depicting the experimental set-up and synthesis of the Cu(DAB) under normal atmospheric conditions: To a stirring solution of 3,3'-diaminobenzidine (DAB) ( $1 \mathrm{~g}, 4.66$ mmoles) in ethanol (a), an equimolar aqueous solution of $\mathrm{Cu}(\mathrm{OAc})_{2} \cdot \mathrm{H}_{2} \mathrm{O}$ (b) was added at room temperature and normal atmospheric pressure to obtain the $\mathbf{C u}(\mathbf{D A B})$ as a deep black precipitate (c). 
Table S1: Vibrational wavenumbers (in $\mathrm{cm}^{-1}$ ) of the bands in the Raman spectrum of DAB

\begin{tabular}{|c|c|}
\hline Raman peaks & $\begin{array}{c}\text { Functional group of DAB } \\
\text { (intensity of the peak) }\end{array}$ \\
\hline 801.95 & $\tau \mathrm{CCCH}(67)\{\gamma \mathrm{CH}\}$ \\
\hline 845.305 & $\tau \mathrm{CCCH}(44)\{\gamma \mathrm{CH}\}, \tau \mathrm{CCCC}(22), \delta \mathrm{CCC}(12)$ \\
\hline 960.59 & $\delta \mathrm{CCC}(45), v \mathrm{CC}(29), v \mathrm{CN}(16)$ \\
\hline 1196.02 & $\delta \mathrm{CCH}(33), v \mathrm{CN}(30), v \mathrm{CC}(24)$ \\
\hline 1314.23 & $\delta \mathrm{CCH}(46), v \mathrm{CC}(32), \delta \mathrm{CNH}(10)$ \\
\hline 1346.48 & $v \mathrm{CC}(38), \delta \mathrm{CCH}(29), \delta \mathrm{CNH}(19)$ \\
\hline 1380.06 & $\tau \mathrm{CC}(57), v \mathrm{CN}(14), \delta \mathrm{CNH}(14)$ \\
\hline 1445.67 & $\delta \mathrm{CCH}(48), \delta \mathrm{CCC}(18), v \mathrm{CN}(14), v \mathrm{CC}(13)$ \\
\hline 1602.40 & $v \mathrm{HNH}(79)\{\mathrm{qNH} 2\}, \tau \mathrm{CCNH}(16)$ \\
\hline \multicolumn{2}{|c|}{$\begin{array}{l}v \text {; stretching, } \delta \text {; in plane bending, } \gamma \text {; out of plane bending, } \tau \text {; torsion, } \rho \text {; scissoring, } r \text {; } \\
\text { rocking, } \omega \text {; wagging, } t \text {; twisting. }\end{array}$} \\
\hline
\end{tabular}




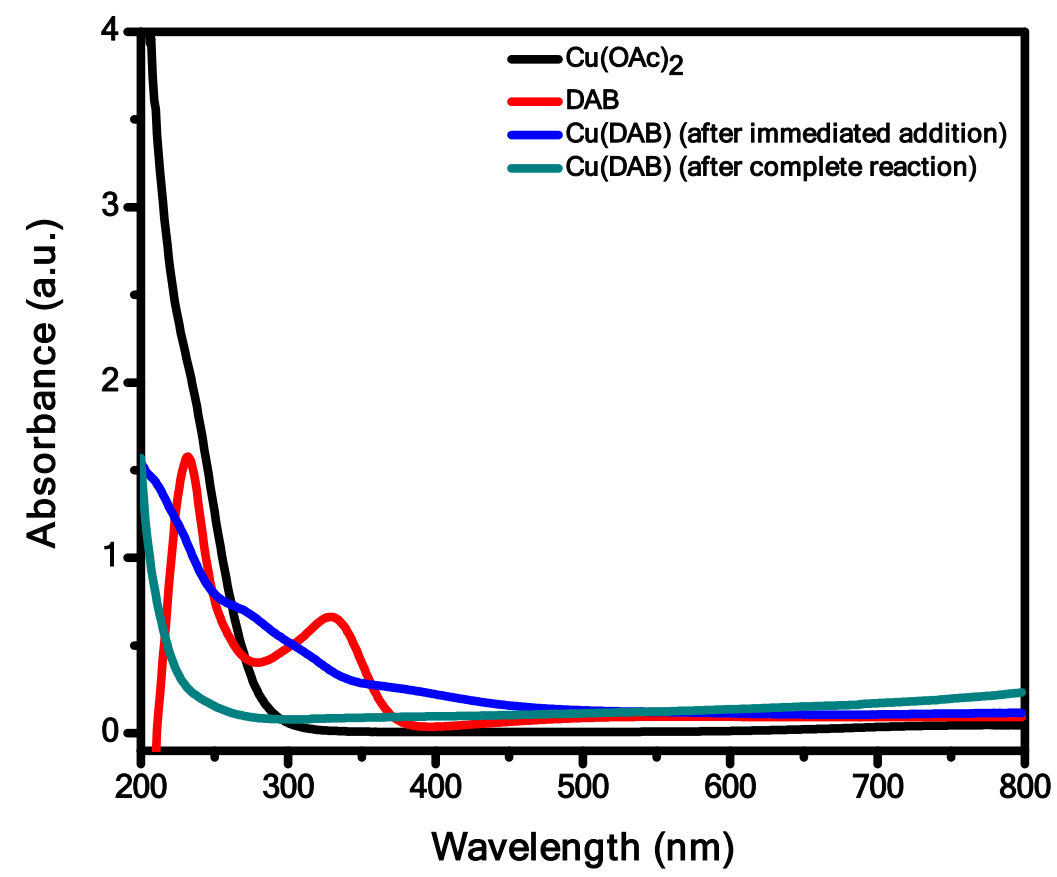

Figure S2: UV-Vis spectra of the reagents used such as $\mathrm{Cu}(\mathrm{OAC})_{2}$ (black trace) and DAB (red trace), and the target COP formed (blue trace) immediately after the initial interaction between the reactants. The nature of blue trace clearly shows the reaction taking place between the $\mathrm{Cu}(\mathrm{OAC})_{2}$ and $\mathrm{DAB}$. The green trace with no spectral pattern resemblance to the black, red \& blue profiles clearly indicate the formation of $\mathrm{Cu}(\mathrm{DAB})$ material. 

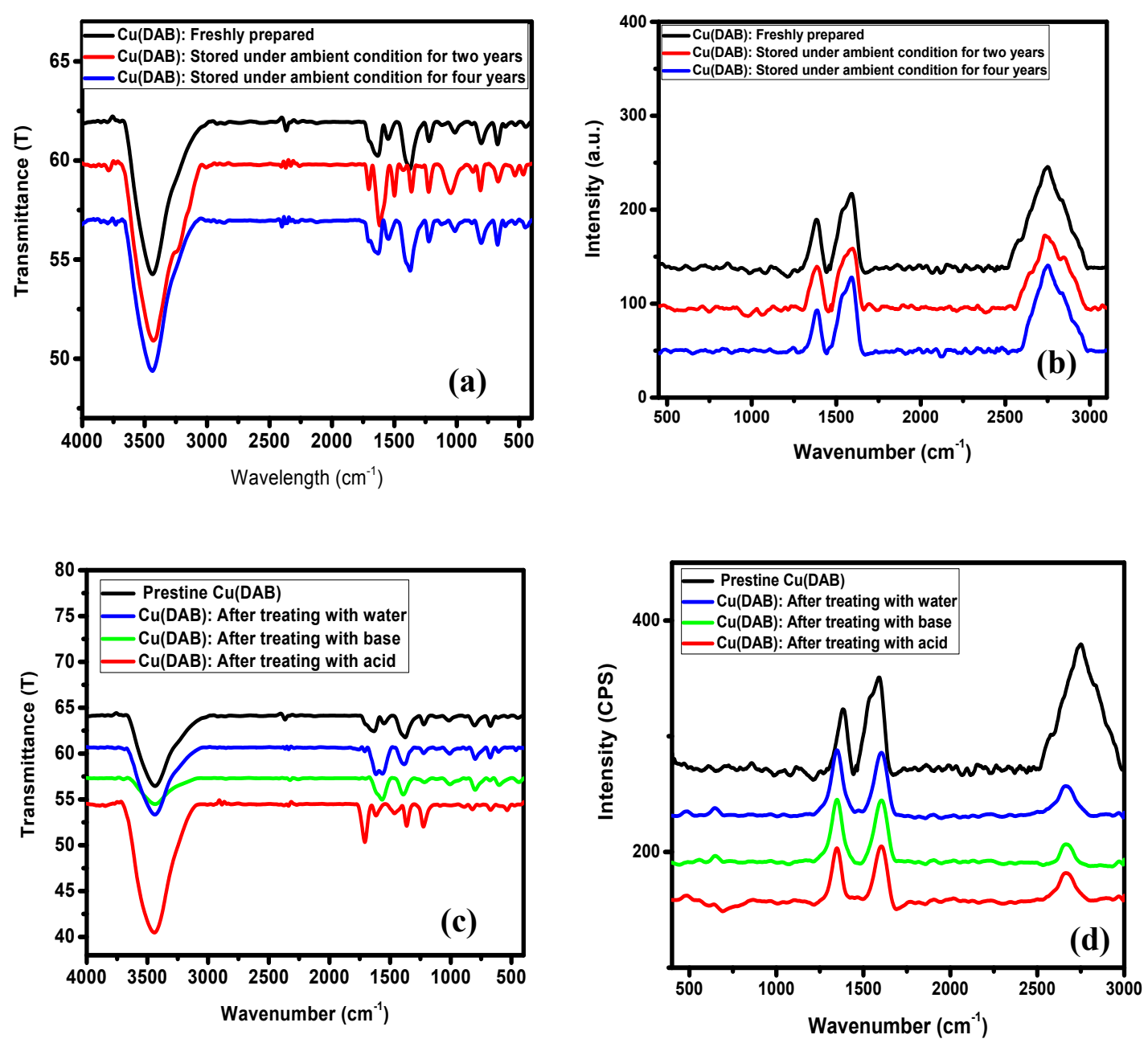

Figure S3: FTIR and Raman spectroscopic profiles depicting the sturdiness of the Cu(DAB) under different storing durations at ambient conditions, and treating with different aqueous solutions. FTIR (a) and Raman (b) spectra of the freshly prepared (black-trace) and those of the $\mathbf{C u}(\mathbf{D A B})$ samples stored under ambient conditions for a longer duration (say 1 to 4 years); here, it may be noted that all the spectral pattern closely match implying the stability of the COP. FTIR (c) and Raman (d) spectra recorded for the pristine sample and the ones treated with water, alkaline (aq) solution and $\mathrm{HCl}(\mathrm{aq})$; as expected, the changes are seen only in the case of sample treated with $\mathrm{HCl}(\mathrm{aq})$ where the protonation may occur. 
Table S2: Results of component analyses of $\mathrm{Cu}(\mathrm{DAB})$ derived from energy dispersive X-ray analysis (EDX) and elemental analysis

\begin{tabular}{|c|c|c|c|}
\hline \multicolumn{2}{|c|}{ Theoretical } & \multicolumn{2}{c|}{ Experimental } \\
\cline { 3 - 4 } \multicolumn{2}{|c|}{} & EDX & Elemental analysis \\
\hline $\mathrm{C}$ & $46.52 \%$ & $48.18 \%$ & $47.58 \%$ \\
\hline $\mathrm{N}$ & $18.08 \%$ & $14.40 \%$ & $16.37 \%$ \\
\hline $\mathrm{H}$ & $4.554 \%$ & - & $4.68 \%$ \\
\hline $\mathrm{O}$ & $10.328 \%$ & $12.95 \%$ & - \\
\hline $\mathrm{Cu}$ & $20.509 \%$ & $24.47 \%$ & - \\
\hline
\end{tabular}




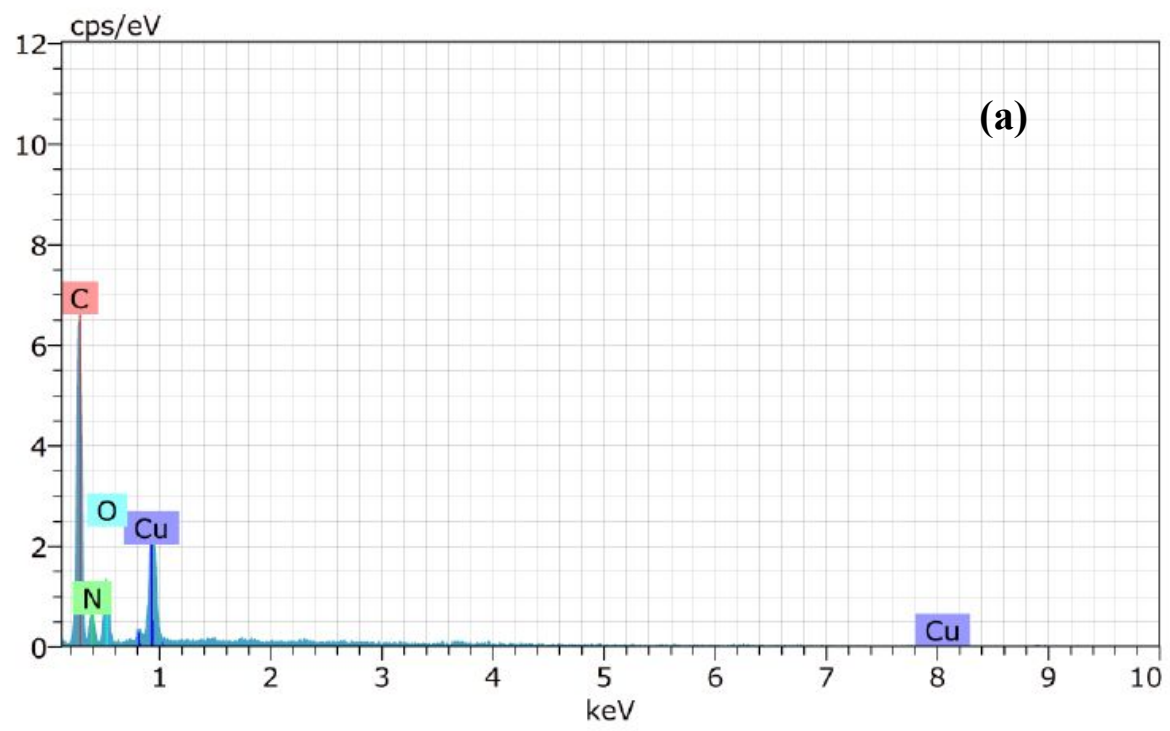

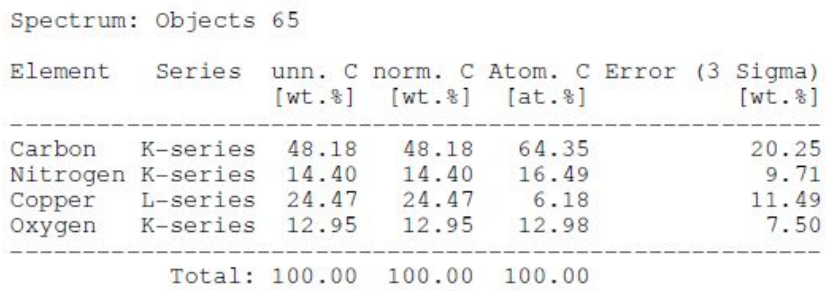

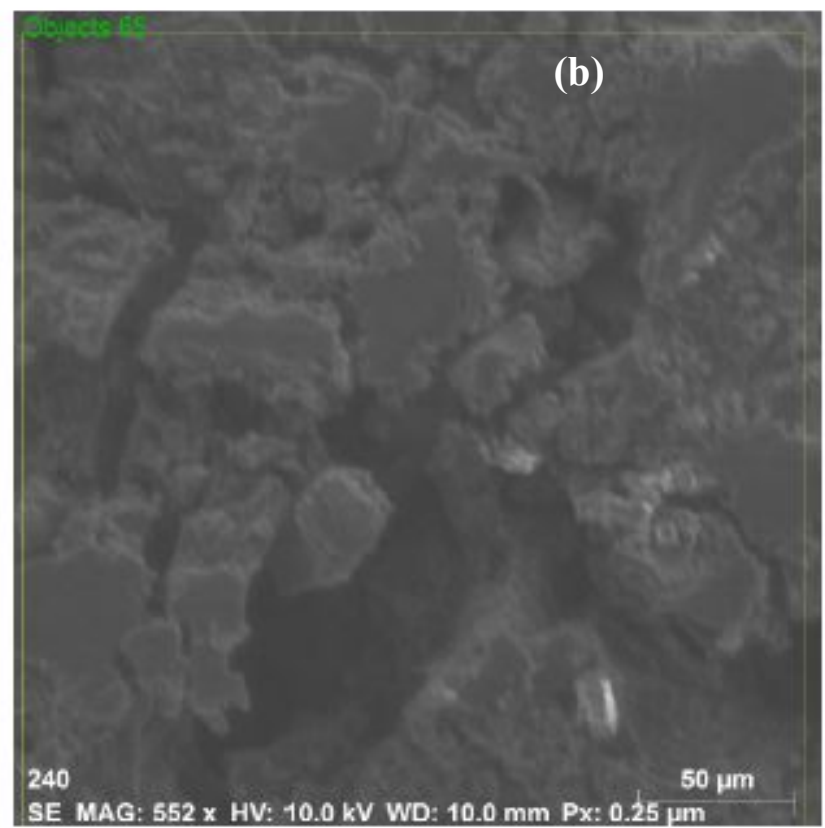

Figure S4. (a) EDX spectrum of the bulk $\mathrm{Cu}(\mathrm{DAB})$ where the atomic percent obtained for $\mathrm{C}$, $\mathrm{N}, \mathrm{Cu}$ and $\mathrm{O}$ respectively is 48.18, 14.40, 24.47 and $12.95 \%$. (b) An image of the portion (yellow marking) of the sample (pellet) subjected for EDX measurement. 

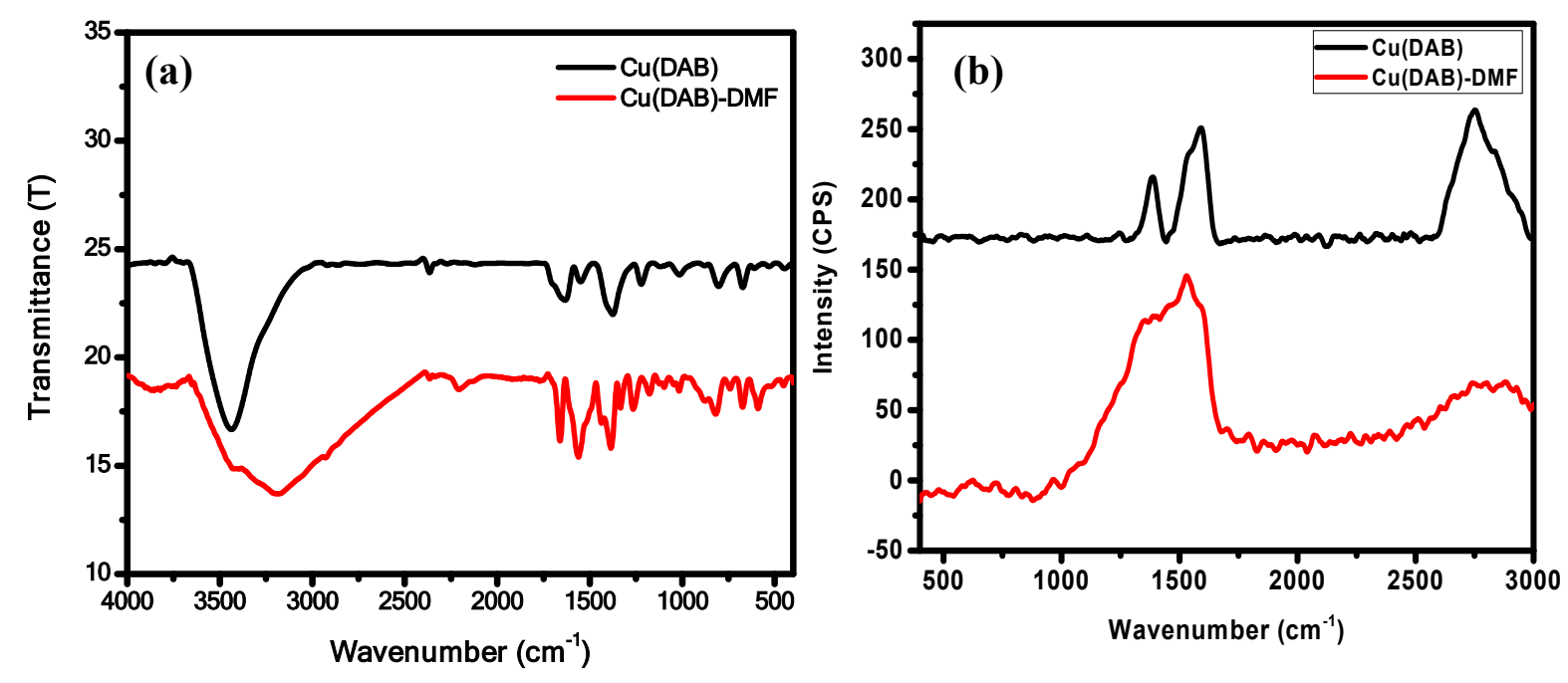

Figure S5: FTIR (a) and Raman (b) spectra of the pristine Cu(DAB) material (black-traces) and the sample collected after dispersing it in DMF (red-traces). The broadening of the peak at around $3000 \mathrm{~cm}^{-1}$ in FTIR spectrum (red-trace) as compared with that of $\mathrm{Cu}(\mathrm{DAB})$ (black tarce) imply the breaking of the $\mathrm{H}$-bond network. Analogously, a notable change seen in the Raman spectrum of the sample treated with DMF (red-trace) as compared to the pristine compound (black-trace) clearly suggest the breaking of $\mathrm{H}$-bondings. 


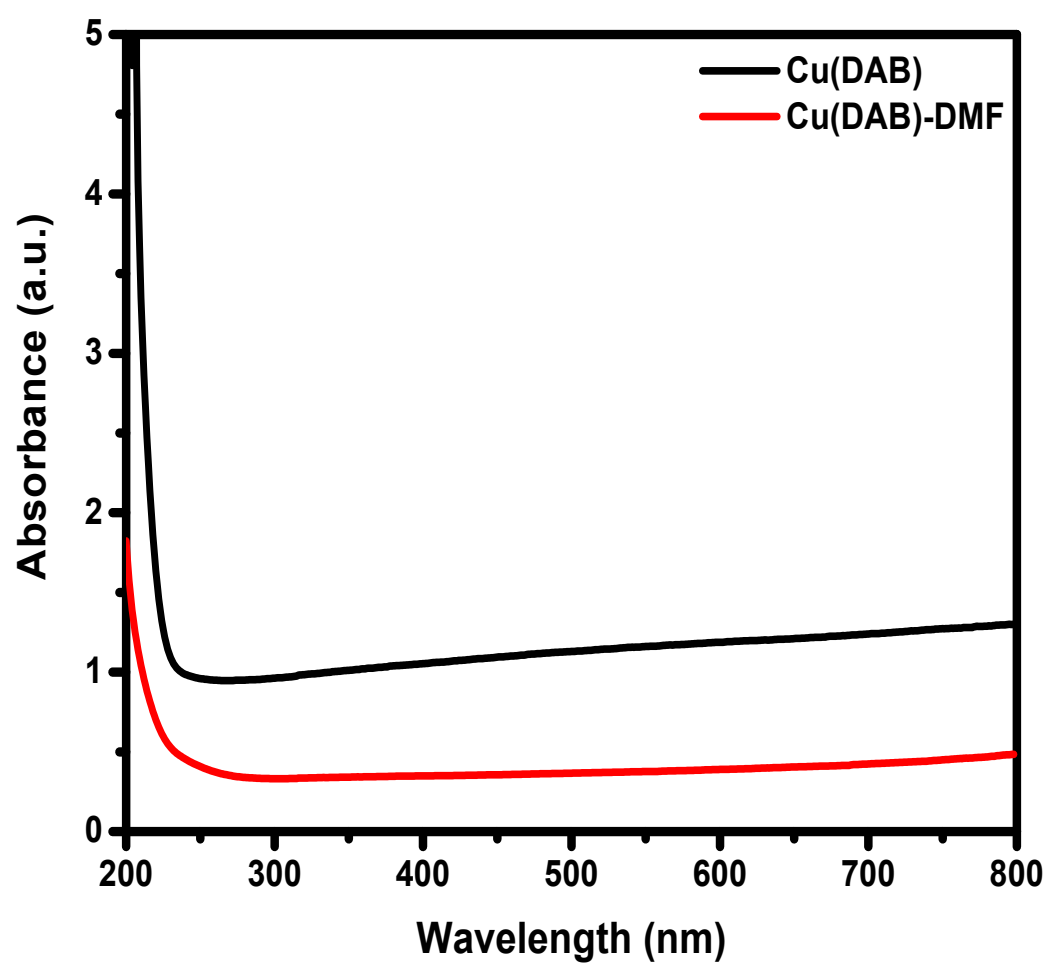

Figure S6: UV-Vis spectra of Cu(DAB) (black trace) and Cu(DAB)-DMF (red trace) dispersed in water. Notice that the onset UV peak $250 \mathrm{~nm}$ of the pristine $\mathrm{Cu}$ (DAB) occurs at $262 \mathrm{~nm}$ when it is treated with DMF; this red-shift of the onset peak can be ascribed to the strong association of $\mathrm{C}=\mathrm{O}$ group of DMF with $\mathrm{N}-\mathrm{H}$ group of COP strands. 

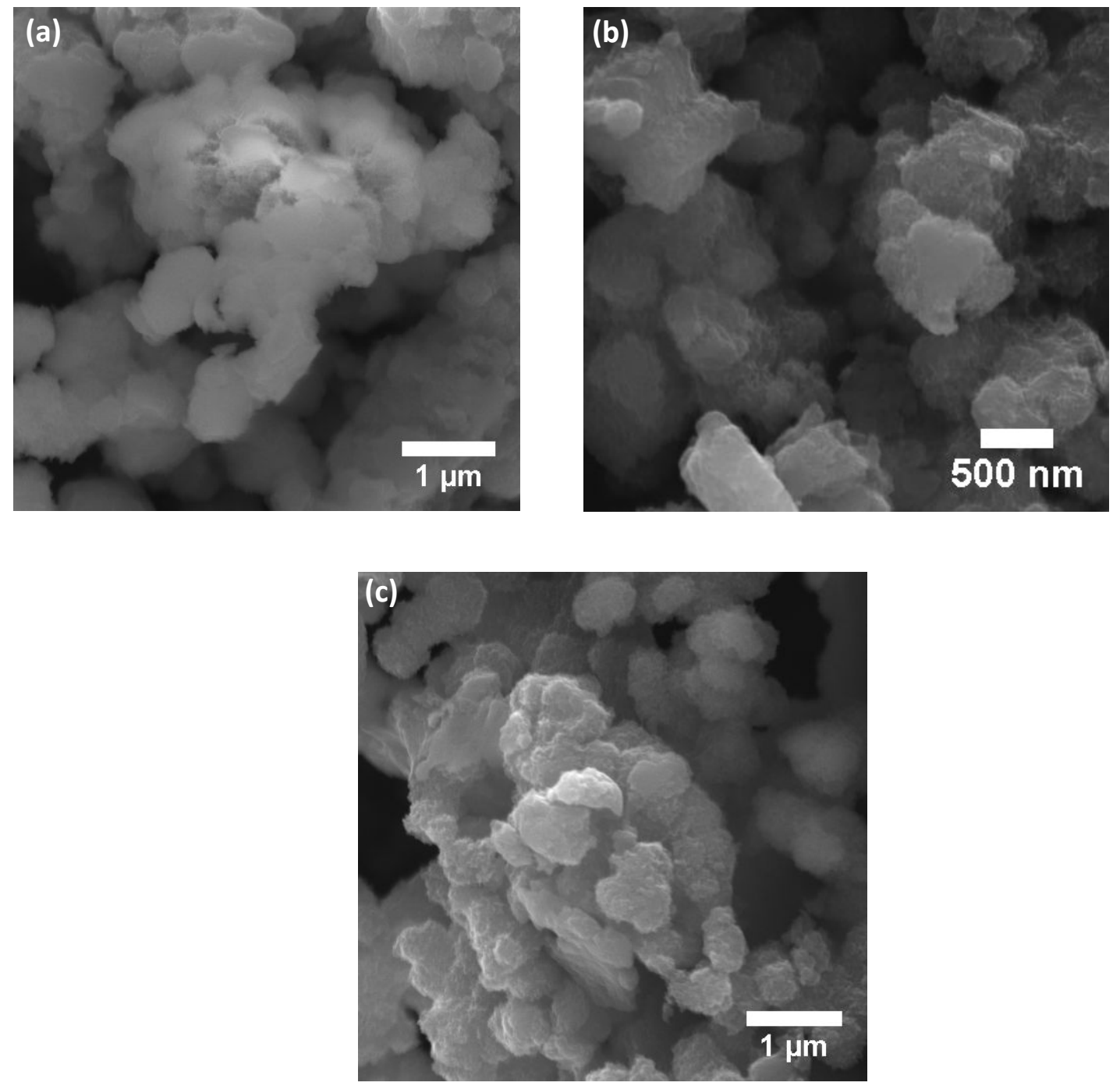

Figure S7: FESEM images of the Cu(DAB) dispersed in DMF and then collected by filtration followed by drying. The smaller sheet size as compared with that of the $\mathrm{Cu}(\mathrm{DAB})$ prepared in aqueous ethanolic media possibly implies the breaking up of $\mathrm{H}$-bonds between 1D COP strands. 


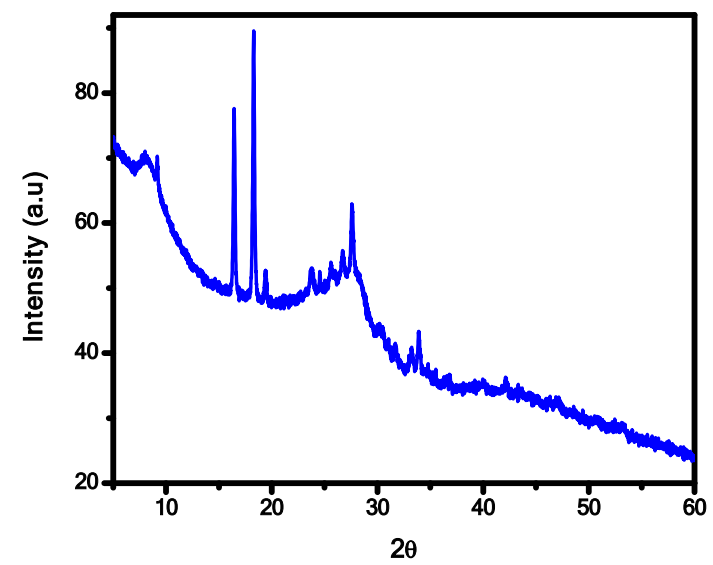

Figure S8: Powder XRD pattern of the pristine Cu(DAB) sample 


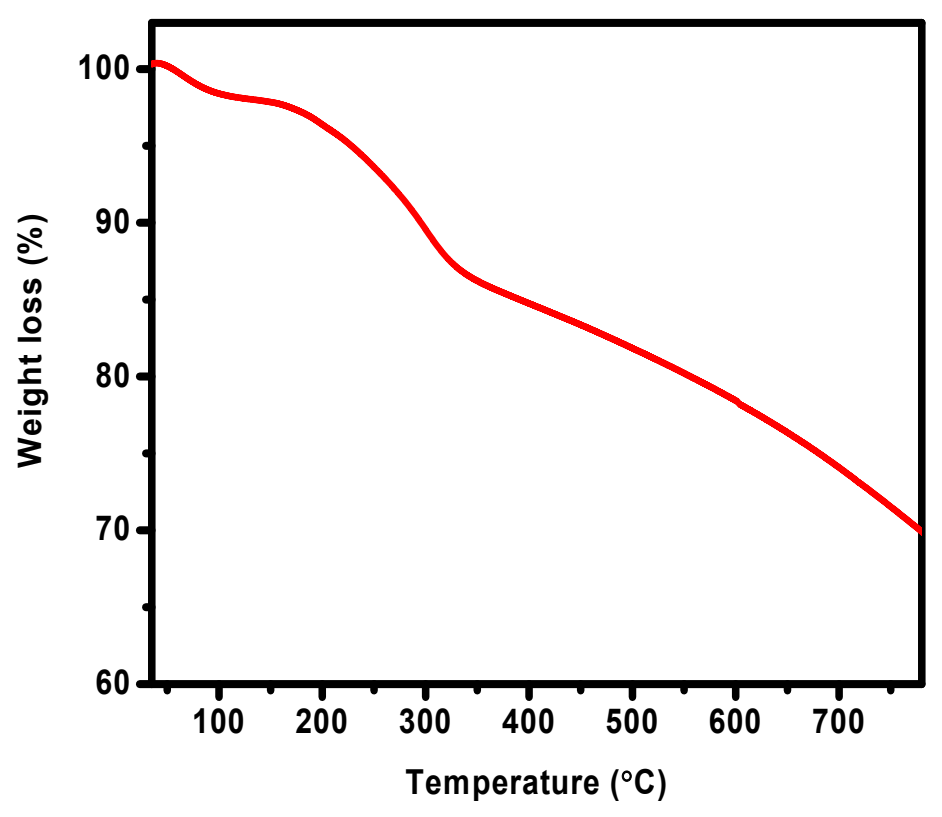

Figure S9: TGA curve of $\mathrm{Cu}(\mathrm{DAB})$. TGA analysis shows the weight loss of $2 \%$ at $90{ }^{\circ} \mathrm{C}$ and 15 $\%$ at $300^{\circ} \mathrm{C}$ which can be respectively attributed perhaps to the loss of water-ethanol traces and slow decomposition of the COP. 
Table S3: Redox data of Cu(DAB)

\begin{tabular}{|l|l|l|l|l|l|l|l|}
\hline $\mathrm{E}_{\mathrm{pc}}(1)(\mathrm{V})$ & $\mathrm{E}_{\mathrm{pa}}(1)(\mathrm{V})$ & $\mathrm{E}_{1 / 2}(1)(\mathrm{V})$ & $\Delta \mathrm{E}_{\mathrm{p}}(\mathrm{mV})$ & $\mathrm{E}_{\mathrm{pc}}(2)(\mathrm{V})$ & $\mathrm{E}_{\mathrm{pa}}(2)(\mathrm{V})$ & $\mathrm{E}_{1 / 2}(2)(\mathrm{V})$ & $\Delta \mathrm{E}_{\mathrm{p}}(\mathrm{mV})$ \\
\hline-0.556 & -0.53 & -0.543 & 26 & -0.039 & -0.11 & -0.084 & 21 \\
\hline
\end{tabular}

$\mathrm{E}_{\mathrm{pc}}-$ Cathodic potential

$\mathrm{E}_{\mathrm{pa}}-$ Anodic potential

$\Delta \mathrm{E}_{\mathrm{p}}-$ Electrochemical band gap 


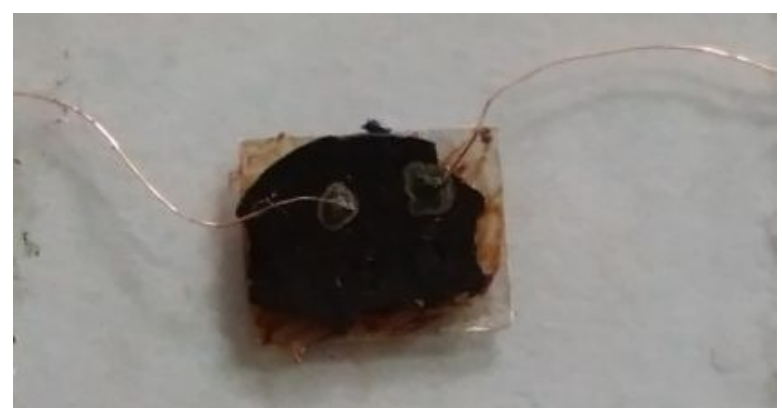

Figure S10: A pellet of $\sim 3 \mathrm{~mm}$ thick with two-probe configuration used for the DC conductivity measurements. 


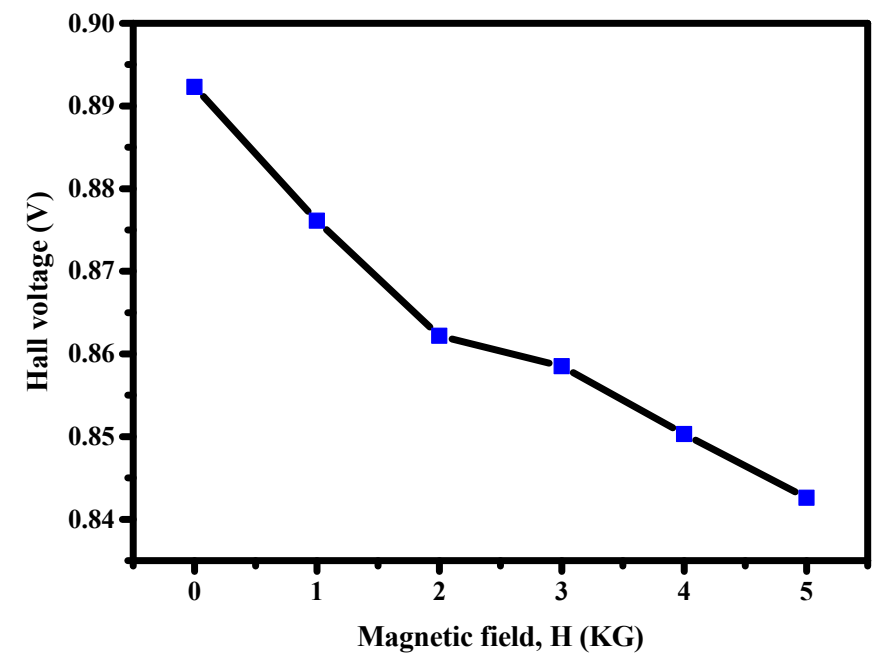

Figure S11: Plot depicting the hall voltage vs applied magnetic field 


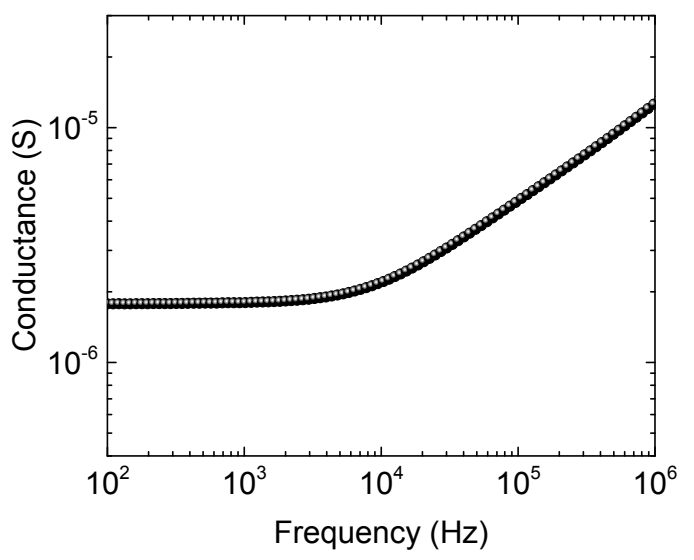

Figure S12: Frequency dependent $A C$ conductivity of $\mathrm{Cu}(\mathrm{DAB})$ 


\section{Determination of effective magnetic moment}

Using the value of Curie constant obtained from the plot (Fig.5), the magnetic moment at high temperature can be calculated using the following equation.

$\chi_{\mathrm{m}}=\mathrm{C} / \mathrm{T}$ (eq. 1)

The Curie Constant $\mathrm{C}$ is equal to $\chi_{\mathrm{m}} \mathrm{T}$ and is proportional to the effective magnetic moment $\mu^{2} \mathrm{eff}$.

$$
\mathrm{C}=\mathrm{N}_{\mathrm{A}} \mathrm{g}^{2} \cdot \mu_{\mathrm{B}}^{2} . \mathrm{J}(\mathrm{J}+1) / 3 \mathrm{~K}_{\mathrm{B}}^{[1]}(\text { eq. } 2)
$$

and effective magnetic moment $\mu_{\text {eff }}=\mathrm{g} \cdot(\mathrm{J}(\mathrm{J}+1))^{1 / 2[2]}$ (eq 3)

from equations 1 and $2 \quad \mu_{\text {eff }}=\left(3 \cdot K_{\mathrm{B}} \cdot \mathrm{C} / \mathrm{N}_{\mathrm{A}}\right)^{1 / 2}$

$=2.84 \mathrm{C}^{1 / 2} \quad$ (eq. 4$)$

Using the above equation 4, the value of $\mu_{\text {eff }}$ is found to be 5.954 .which is close to the spin only value of four uncoupled $\mathrm{Cu}(\mathrm{II})$ ions having $\mathrm{S}=1 / 2$ and $\mathrm{g}=2$ which is 5.910 . 


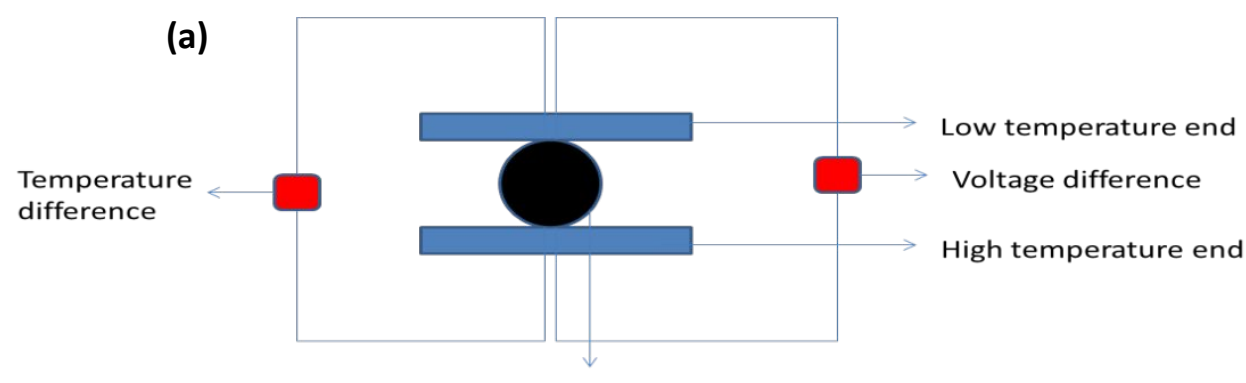

Cu.DAB pellet
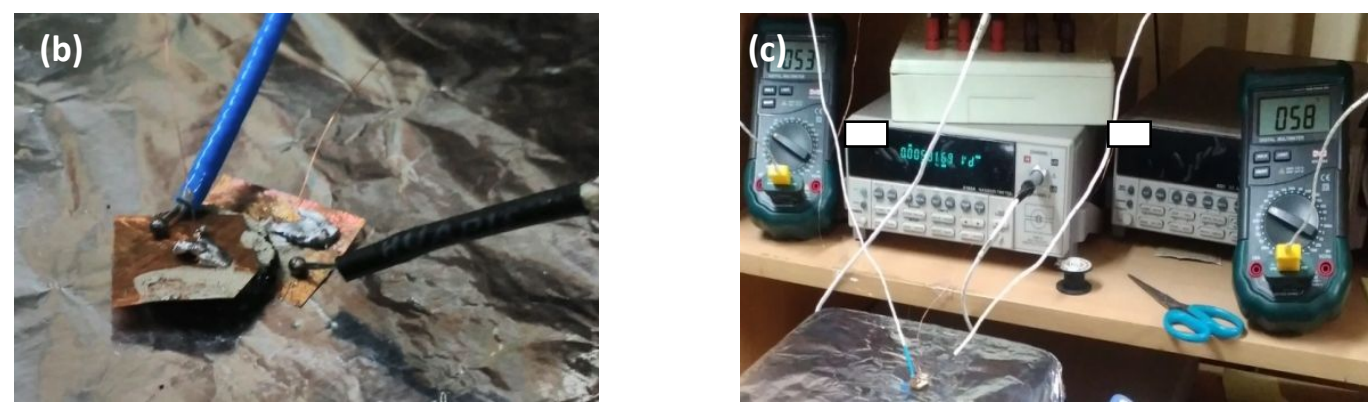

Figure S13: (a) Schematic diagram showing the two point configuration set-up used for thermoelectric measurement, (b) photograph of a homemade two-point geometry set-up employed for the experiment and (c) photograph of the entire experimental set-up used 


\section{References}

1. Kittel, Charles. Introduction to Solid State Physics, 8th Edition. Wiley. p. 304

2. Buschow, K.H.J; de Boer, F.R. Physics of Magnetism and Magnetic Materials, Springer 